\title{
The investigation of the incidence rate of bradycardia caused by nasopharynx irritation and its related factors in adenoidectomy surgery
}

\author{
NADER SAKI ${ }^{1, A, B}$, AHMAD EBADI 2, B, D, SHAHRAM RAJAEI BEHBAHANI ${ }^{3, B, C}$, \\ SOHEILA NIKAKHLAGH ${ }^{1, A, B}$, MINA AKBARPOUR ${ }^{4, E, F}$
}

\author{
${ }^{1}$ Hearing Research Center, Ahvaz Jundishapur University of Medical Sciences, Ahvaz, Iran \\ ${ }^{2}$ Department of Cardiac Anesthesiology, Atherosclerosis Research Center Golestan Hospital, and Pain Research \\ Center, Ahvaz Jundishapur University of Medical Sciences, Ahvaz, Iran \\ ${ }^{3}$ Department of Pediatrics, Golestan Hospital, Ahvaz Jundishapur University of Medical Sciences, Ahvaz, Iran \\ ${ }^{4}$ Student Research Committee, Ahvaz Jundishapur University of Medical Sciences, Ahvaz, Iran
}

A - Study Design, B - Data Collection, C - Statistical Analysis, D - Data Interpretation, E - Manuscript Preparation, F - Literature Search, G - Funds Collection

Summary Background. Adenoidectomy is the most common way to stimulate the vagus nerve, and ultimately bradycardia. Objectives. The aim of this study was to determine the incidence rate of bradycardia caused by nasopharynx irritation and its related factors in patients undergoing adenoidectomy surgery.

Material and methods. The current study is a descriptive-analytic cross-sectional study. 142 children under adenoidectomy surgery enter the study in 2017. Demographic data, hemoglobin, echocardiography, $\mathrm{S}_{\mathrm{p}} \mathrm{O}_{2}$, heart rate of patient before and after anesthesia and during and after surgery, duration of surgery, use or non-use of atropine and its amount were collected. Sampling was carried out using the sequential non-probabilistic method. SPSS version 22 was used to analyze the data.

Results. The average age was 3.1 years. The rate of bradycardia among 1 to 2 -year-old patients was $38.8 \%$ (14 patients) and among 2 to 6 -year-old patients it was $12.4 \%$ (13 patients). The results of 1 to 2 -year-old patients showed a significant relationship between bradycardia and age. The results of 2 to 6 -year-old patients showed a decrease in bradycardia as age increases to 6 years. There was a significant relationship between bradycardia and $\mathrm{S}_{\mathrm{p}} \mathrm{O}_{2}$ rate at the ages of 1 to 2 years and 2 to 6 years. With bradycardia, the patient $\mathrm{S}_{\mathrm{p}} \mathrm{O}_{2}$ dropped. There was a significant relationship between bradycardia and duration of surgery, and also atropine use.

Conclusions. Due to the occurrence of early bradycardia in early ages in adenoidectomy and the possibility of affecting the cardiac output, more precision during this surgery by anesthesiologists and surgeons leads to a reduction in adverse bradycardia effects on the heart.

Key words: adenoidectomy, anesthesia, bradycardia, atropine, child.

Saki N, Ebadi A, Behbahani SR, Nikakhlagh S, Akbarpour M. The investigation of the incidence rate of bradycardia caused by nasopharynx irritation and its related factors in adenoidectomy surgery. Fam Med Prim Care Rev 2018; 20(2): 167-170, doi: https://doi. org/10.5114/fmpcr.2018.76462.

\section{Background}

Adenoids or pharyngeal tonsils are a triangular mass of lymphoid tissue which are located in the posterior part of the nasopharynx. They are a part of the body's immune system against infections. They are formed during the third to seventh embryonic months and exist at birth. During the first few weeks after birth, they are colonized by bacteria. Gradually, the texture degenerates and loses its function in adulthood. At the age of 2 to 6 they may be inflamed by viral infections and cause difficulty in breathing, especially when sleeping [1-5]. Swollen adenoids can cause obstruction of the Eustachian tube and, consequently, secondary otitis media, which can lead to hearing loss $[2,3]$. Adenoidectomy indications include obstruction of the esophagus, adenoid hypertrophy, severe snoring, recurrent chronic sinusitis, secondary chronic puerperal rhinitis to the chronic adenoiditis, and patients with hypo-nasal speech $[3,5]$.

Bradycardia is heart rate less than normal [4]. Bradycardia is referred to as heart rate less than 100 beats per minute in infants below one year, less than 90 beats per minute in one to two-year-old children, less than 80 beats per minute in two to five- -year-old children and less than 70 per minute in six-years-old children. The most common cause of bradycardia is hypoxemia $[6,7]$. The heart echo is a widespread and non-invasive approach in which sound waves harmless for humans determine the image of the heart's components and the rate of blood flow. Using this method, a detailed look at the walls of the heart, the valves, and the beginning of the large arteries can be achieved. The non-invasiveness of this test is one of the special advantages of it $[8,9]$.

Adenoidectomy is performed under general anesthesia. The drugs used in general anesthetics include inhaled drugs, non-opioid intravenous drugs, opioid intravenous drugs and muscle relaxants [8].

Regarding the type of anesthesia, and due to the role of pharyngeal nerves and irritability of the nasopharynx and nerves 9 and 10 , as well as the possibility of changes in heart rate when manipulating the throat, considering some points during adenoid surgery is necessary. Paying attention to the patient's pulse during surgery is an important point for patients during surgery $[9,10]$.

Since adenoidectomy is a very common surgery, if the presence of bradycardia is proved during surgery and anesthesia and the use or non-use of atropine and its value, the importance of 
paying attention to specific points, and more precision during this surgery by anesthesiologists and surgeons is determined. This reduces bradycardia and the adverse effects of it $[10,11]$.

In a study by Philly and colleagues in 2007 on 101 patients during facial palatal cleft surgery, the apparent decrease in heart rate was occasionally shown to be $40 \%$. In this study, the anesthetic method was the same for all patients [9]. In a study by Vitanen and a colleague, Gerald Bar, in 1999, the effects of halothane and levoflurane on bradycardia were compared during adenoidectomy. In this study, 701 to 3 -year-old children were studied. Heart rate below 70 was considered as bradycardia. Bradycardia occurred with halothane $15 \%$ and with sevoflurane 6\% [8].

A study by Kinsella and Tuckey in 2001 noted bradycardia presence during surgery and general anesthesia. However, the percentage of bradycardia occurrence and its relationship with age and gender are not mentioned [10]. In a study by Ebadi and his colleagues in 2007, bradycardia presence was reported during adenoidectomy. Bradycardia was reported according to age and gender in girls (38.7\%) and in boys (39.53\%) [11].

\section{Objectives}

Considering the importance of this issue, we decided to study the percentage of bradycardia occurrence during adenoidectomy surgery, and its association with age, gender, heart rate, duration of surgery, use or non-use of atropine and its amount.

\section{Material and methods}

\section{Study design}

This study is a descriptive-analytic cross-sectional study. This is a randomized clinical study. The research population included girls and boys under the age of six, who underwent adenoidectomy surgery in Imam Khomeini and Apadana hospital, Ahvaz, from 21/06/2016 to 23/07/2017 (one year). This research was the result of an MD thesis approved by the Ethical Committee of Ahvaz Jundishapur University of medical sciences, Ahvaz, Iran.

\section{Setting, participants and variables}

The criteria for entering this study include having the required indications for adenoidectomy surgery and age less than 6. Patients who had heart disease or bradycardia before surgery were excluded from the study.

In this study, the same location, type of surgery, surgeon, anesthetic and anesthetic methods were selected. Anesthetics include Thiopental, Atracurium, Pethidine and Acetaminophen suppository. The type of anesthetic was kept constant for all patients. Bradycardia is not a side effect of the chosen medications. A questionnaire, containing demographic information such as age, gender, history of URTI, patient's hemoglobin, echocardiography status, $\mathrm{S}_{0} \mathrm{O}_{2}$, heart rate before and after anesthesia and during surgery (using curette) and after the completion of surgery, duration of surgery, use or non-use of atropine and its amount, was prepared and completed.

\section{Statistical analyses}

For data analysis, the descriptive statistics methods, including frequency distribution tables, graphs and central indices, and appropriate dispersion such as mean and standard deviation were used to describe the studied variables. The incidence rate and $95 \%$ confidence interval were also calculated.

Normality of quantitative data was checked by the Kolmogorov-Smirnov test. The chi-square test was used to de- termine the relationship between qualitative variables, and the independent $t$-test or nonparametric equation was used to compare quantitative values between two groups. The significance level of the tests was considered to be less than 0.05 . Data analysis was done using SPSS software 23.

\section{Results}

\section{Descriptive data}

The results of this study showed that $59.2 \%$ ( 84 individuals) were boys and $40.8 \%$ (58 individuals) were girls. The average age of 142 patients was 3.1 years, and the minimum and maximum age of these patients was 1 and 5.9 years, respectively (Table 1).

\begin{tabular}{|l|l|l|}
\hline \multicolumn{4}{|l|}{ Table 1. Age distribution of patients } \\
\hline Age & $n$ & $\%$ \\
\hline $1-2$ & 37 & 26.1 \\
\hline $2-3$ & 18 & 12.7 \\
\hline $3-4$ & 53 & 37.3 \\
\hline $4-5$ & 28 & 19.7 \\
\hline$>5$ & 6 & 4.2 \\
\hline Total & $\mathbf{1 4 2}$ & $\mathbf{1 0 0 . 0}$ \\
\hline
\end{tabular}

\section{Outcome data and main results}

In this study, the rate of bradycardia among 1 to 2 -year-old patients was $38.8 \%$ (14 patients). The results of the study showed that there is a significant relationship between bradycardia and age in 1 to 2 -year-old patients. As the age goes up to 2 years, bradycardia increases ( $p$-value $=0.013$ ). There is also a significant relationship between bradycardia and $\mathrm{S}_{\mathrm{p}} \mathrm{O}_{2}$, rate in the ages of 1 to 2 years. With bradycardia, the patient experiences a drop in $\mathrm{S}_{p} \mathrm{O}_{2}(p$-value $=0.005)$. There was a significant relationship between bradycardia and duration of surgery. In patients with bradycardia, the duration of surgery increased ( $p$-value $=0.05)$. There was a significant relationship between the incidence of bradycardia and the use of atropine. In patients with atropine, there was a lower bradycardia ( $p$-value $=0.03$ ). The results also showed that there was no significant relationship between bradycardia and gender ( $p$-value $=0.13$ ). In this study, the bradycardia rate was $12.4 \%$ (13 patients) among 2 to 6 -year-old patients (Table 2). The results of 2 to 6 -year-old patients showed a significant relationship between bradycardia and age. Bradycardia decreases as age increases to 6 years ( $p$-value $=0.03)$. There was a significant relationship between bradycardia and hemoglobin level. Low hemoglobin level decreases bradycardia ( $p$-value $=0.03$ ). There was a significant reverse relationship between bradycardia and surgery duration. In patients with bradycardia, the duration of surgery increased $(p$-value $=0.008)$. There was a significant relationship between bradycardia and $\mathrm{S}_{p} \mathrm{O}_{2}$ level at the age of 2 to 6 years. With the incidence of bradycardia in patients, $\mathrm{S}_{\mathrm{p}} \mathrm{O}_{2}$ decreased ( $p$-value $=$ $0.01)$. However, there was no significant relationship between bradycardia and gender ( $p$-value $=0.13$ ) (Table 3). Table 4 shows the variation of echocardiographic changes.

\begin{tabular}{|l|l|l|}
\hline \multicolumn{3}{|l|}{ Table 2. Bradycardia in different ages } \\
\hline Age & Pulls (0-89) & Pulls (>90) \\
\hline $1-2$ & 14 & 23 \\
\hline $6-2$ & 13 & 92 \\
\hline Total & $\mathbf{2 7}$ & $\mathbf{1 1 5}$ \\
\hline
\end{tabular}




\begin{tabular}{|c|c|c|c|}
\hline & & $\begin{array}{l}\text { Bradycardia } \\
1-2 \text { years } \\
\end{array}$ & \begin{tabular}{|l|}
$\mathrm{S}_{\mathrm{p}} \mathrm{O}_{2}$ \\
$1-2$ years \\
\end{tabular} \\
\hline \multirow[t]{3}{*}{$\begin{array}{l}\text { Bradycardia } \\
1-2 \text { years }\end{array}$} & $\begin{array}{l}\text { Spearman's } \\
\text { rho Correlation }\end{array}$ & 1 & 0.541 \\
\hline & Sig. (2-tailed) & & 0.005 \\
\hline & $n$ & 37 & 37 \\
\hline
\end{tabular}

\begin{tabular}{|c|c|c|c|}
\hline \multicolumn{4}{|c|}{ Cardiac disease } \\
\hline & & $n$ & $\%$ \\
\hline \multirow[t]{10}{*}{ Valid } & normal & 99 & 69.7 \\
\hline & MVP & 12 & 8.5 \\
\hline & $\bmod T R$ & 8 & 5.6 \\
\hline & mild PI & 8 & 5.6 \\
\hline & mild TR & 4 & 2.75 \\
\hline & mild LVH & 4 & 2.75 \\
\hline & mild-mod TR & 3 & 2.2 \\
\hline & $\mathrm{COA}$ & 2 & 1.4 \\
\hline & mild LVE & 2 & 1.4 \\
\hline & Total & 142 & 100.0 \\
\hline
\end{tabular}

\section{Discussion}

Adenoidectomy and adenotonsillectomy are among the most common types of surgeries performed in children. The rate of surgery in the United States and Britain is approximately 50 and 65 individuals among a population of 10,000 people, respectively. In the Netherlands, the number of surgeries in 1998 was 115 , which was even higher than previous decades $[10,18]$. Among ear, nose and throat surgeries, adenotonsillectomy is the most common method that directly stimulates the vagus nerve, and ultimately bradycardia [11]. In children, heart rate is an important factor for cardiac output because the growing heart has lower capacity, contraction and impact volume, and there cannot be much increase. Therefore, when bradycardia occurs in children during anesthesia, cardiac output decreases and may lead to serious cardiac arrhythmias, and even heart failure $[12,13]$.

\section{Interpretation and generalizability}

The study of Saki, Ebadi and colleagues in Iran in 2009 on 747 patients with adenoidectomy, comprising $74 \%$ (554) boys and $26 \%$ (193) girls, is consistent with the present study. Our study also showed that there was no significant relationship between bradycardia and gender [11, 13]. In the study of Ebadi and colleagues, there were $29.8 \%$ ( 223 people) 0 to 3 -year-old individuals and $27 \%$ (202 people) 3 to 6 -year-old individuals [11]. Like the current study, the age group of 2 to 6 was predominant.

Normal heartbeat decreases with increasing age in children. Therefore, heart rate less than 100 pulses per minute in lowage children is considered as bradycardia. In 3-year-old children, heart rate less than 65 pulses per minute is considered as bradycardia. Even in early days of birth, the risk of bradycardia is higher due to imbalance in autonomic nerves $[10,14]$.

In the present study the rate of bradycardia among 1 to 2-year-old patients was $38.8 \%$ (14 patients) and among 2 to 6 -year-old patients it was $12.4 \%$ (13 patients). The results of 1 to 2-year-old patients showed a significant relationship between bradycardia and age. Bradycardia increases as age increases to 2 years. The results of 2 to 6 -year-old patients showed a decrease in bradycardia as age increases to 6 years.

In the study of Murat and collogues, the rate of bradycardia among children under one year was $0.32 \%$ (12 out of 3681 ). It was $0.07 \%$ (9 out of 12495) among 1 to 7 -year-old children [13].

In the study of Ebadi and colleagues, 331 cases of bradycardia were reported out of 747 patients. The rate of bradycardia among children under 3 years of age was $21.9 \%$ (164 cases) and among 3 to 6 -year-old children it was $13.7 \%$ (103 cases) [11].

In the study of Keenan and colleagues, 84 cases of bradycardia were reported out of 7979 patients. The rate of bradycardia among 0 to 1-year-old children was $1.27 \%$ (59 cases out of 4645 children), among 1 to 2 -year-old children it was $0.98 \%$ ( 19 cases out of 1932 children), among 2 to 3 -year-old children it was $0.65 \%$ ( 5 cases out of 774 children), and among 3 to 4 -year-old children it was $0.16 \%$ ( 1 case out of 628 children) [14]. These studies are in line with our study.

During general anesthesia children are at risk of bradycardia due to hypoxemia or hypervagotomy. Hypoxemia can be caused by the prescription of anesthetic drugs or other drugs. Bradycardia associated with anesthesia may lead to inadequate cardiac output and oxygen supply to vital organs $[12,15]$.

In our study there was a significant relationship between bradycardia and $\mathrm{S}_{\mathrm{p}} \mathrm{O}_{2}$ level in the ages of 1 to 2 years and 2 to 6 years. With bradycardia, the patients' $\mathrm{S}_{\mathrm{p}} \mathrm{O}_{2}$ dropped.

In the study of Murat and collogues, the rate of bradycardia among children under one year was $0.32 \%$ (12 out of 3681). It was $0.07 \%$ (9 out of 12495) among 1 to 7 -year-old children. Based on this study, it can be said that considering the age range and high bradycardia and hypoxia levels in different age intervals, higher a bradycardia rate brings about a higher hypoxemia rate. Therefore, this study is in line with our study [13].

In our study there was a significant relationship between bradycardia and hemoglobin level. With the reduction in hemoglobin, bradycardia reduces. However, there has been no accurate study to show the relationship between bradycardia and hemoglobin. The study of Gunawardana and colleagues (1999) was performed on 200 3-month-old to 5-year-old children with hemoglobin of more than 7 under general anesthesia and cleft palate surgery. In this study, 8 individuals (out of 68) having 7 to 10 hemoglobin level and 6 individuals (out of 132) with hemoglobin more than 10 had hypoxia and, consequently, transient bradycardia. This study showed that a person with low hemoglobin is more likely to have hypoxia and, consequently, bradycardia during induction of anesthesia and surgery. Therefore, this study was not consistent with our study $[13,15]$.

In our study, there was a significant relationship between bradycardia and duration of surgery. In patients with bradycardia the duration of surgery increased.

The study of Keenan and colleagues investigated the duration of surgery (non-cardiac surgery) in 0 to 1 -year-old children with bradycardia, which was significant. Among 4645 patients under anesthesia, 378 had a surgery duration of more than 4 hours. $3.7 \%$ (14 people) of them had bradycardia. 4267 patients had a surgery duration of less than 4 hours. 1/05\% (45 people) had bradycardia. Therefore, this study was in line with our study [14].

As previously discussed, children who require intracheal intubation usually have bradycardia. There is a discussion on the effects of pretreatment with atropine in children under the age of 5 years [15]. With the prescription of atropine or other anticholinergic drugs, the incidence of bradycardia can be reduced. At low doses of atropine there is the possibility of bradycardia occurrence. In excessive use of atropine ventricular dysrhythmia and tachyarrhythmia may occur $[15,16]$. Most evidence of atropine use as standard in all children under the age of 18 was gathered before 1990. Given the risks and side effects of atropine, it cannot be recommended as a standard pretreatment [15].

In our study there was a significant relationship between the incidence of bradycardia and the use of atropine. Patients who used atropine had lower bradycardia.

In the study of Mirakhur regarding the pretreatment of atropine in children, this relationship was also meaningful. The use of atropine prior to induction of anesthesia reduced bradycardia in patients [16]. 
Fastle and collogues (2004) showed that atropine was not used routinely in children before intubation. Pretreatment of atropine does not prevent all bradycardia in children. However, using it can reduce bradycardia before intubation [17].

\section{Limitations of the study}

This study was done in one city and just one center, and we did not have access to a larger pool from which to select patients, and also a bigger sample size, so we designed this study just for the above-mentioned patients.

\section{Conclusions}

The results of this study can provide evidence of adenoidectomy care and the use of medication to control bradycardia, and evaluate the patient in terms of cardiovascular state before adenoidectomy to prevent bradycardia. In our study there was a significant relationship between the incidence of bradycardia and the use of atropine. Patients who used atropine had lower bradycardia. More precision during this surgery by anesthesiologists and surgeons leads to a reduction in adverse bradycardia effects on the heart.

Acknowledgments. The authors would like to thank the research deputy of Ahvaz Jundishapur University of medical sciences, Ahvaz, Iran for their valuable cooperation. This research was the result of an MD thesis (Approved by the Ethical Committee of Ahvaz Jundishapur University of medical sciences).

Source of funding: Ahvaz Jundishapur University of Medical Sciences, Ahvaz, Iran. Conflicts of interest: The authors declare no conflicts of interest.

\section{References}

1. Ramos SD, Mukerji S, Pine HS. Tonsillectomy and adenoidectomy. Pediatr Clin North Am 2013; 60(4): 793-807.

2. Kretzschmar MJ, Siccama I, Houweling PL, et al. Hypoxemia and bradycardia in children during adenotonsillectomy without intubation. Ned Tijdschr Geneesk 2010; 154: 1715-1719.

3. Saki N, Nikakhlagh S, Hekmatshoar M, et al. Evaluation of hearing results in otosclerotic patients after stapedectomy. Iran J Otorhinolaryngol 2011; 23(65): 127-133.

4. Tuli BS, Tuli IP, Singh AD, et al., eds. Textbook of ear, nose, and throat. New Delhi: Jaypee Brothers; 2005.

5. Kour J, Ahmed JA, Nashiruddullah N, et al. Electrocardiographic interpretation of the nasopharyngeal response in conscious rabbits. IJAR 2016; 50(6): 900-904.

6. Bayat A, Pourbakht A, Saki N, et al. Vestibular rehabilitation outcomes in the elderly with chronic vestibular dysfunction. Iran Red Crescent Med J 2012; 14(11): 705-708.

7. Toprak V, Yentur A, Sakarya M. Anasthetic management of sever bradycardia during general anesthesia using temporary cardiac pacing. BrJ Anaesth 2002; 89(4): 655-657.

8. Viitanen $\mathrm{H}$, Baer $\mathrm{G}$, Koivu $\mathrm{H}$, et al. The hemodynamic and holter ECG changes during Halotan 7 sevoflurance anesthesia for adenoidectomy. Anesth Analg 1999; 87(6): 1423-1425.

9. Philly T. Bradycardia during cleft palate surgery. BJORL 2007: 451.

10. Kinsella SM, Tuckey JP. Perioperative bradycardia and asystole. Br J Anaesth 2002; 86(6): 859-868.

11. Ebadi A, Saki N, Nikakhlagh S, et al. Adenoidectomy-induced bradycardia in anesthetized children. Eur J Dent Med 2009; 1: 8-15, doi: 10.3923/ejdm.2009.8.15.

12. Lammers RL, Willoughby-Byrwa M, Fales WD. Errors and error-producing conditions during a simulated, prehospital, pediatric cardiopulmonary arrest. Simul Healthc 2014; 9(3): 174-183.

13. Murat I, Constant I, Maud'huy H. Perioperative anaesthetic morbidity in children: a database of 24,165 anesthetics over a 30-month period. Paediatr Anaesth 2004; 14(2): 158-166.

14. Keenan RL, Shapiro JH, Kane FR, et al. Bradycardia during anesthesia in infants. An epidemiologic study. Anesthesiology 1994; 80: 976-982.

15. Gunawardana RH, Gunasekara SW, Weerasinghe JU. Anesthesia and surgery in pediatric patients with low hemoglobin values. Indian $J$ Pediatr 1999; 66(4): 523-526.

16. Mirakhur RK. Premedication with atropine or glycopyrrolate in children. Effects on heart rate and rhythm during induction and maintenance of anesthesia. Anaesthesia 1982; 37(10): 1032-1036.

17. Fastle RK, Roback MG. Pediatric rapid sequence intubation incidence of reflex bradycardia and effects of pretreatment with atropine. Pediatr Emerg Care 2004; 20(10): 651-655.

18. Nikakhlagh S, Rahim F, Boostani H, et al. The effect of adenotonsillectomy on quality of life in adults and pediatric patients. Indian J Otolaryngol Head Neck Surg 2012; 64: 181-183.

Tables: 4

Figures: 0

References: 18

Received: 11.03.2018

Reviewed: 20.03.2018

Accepted: 06.04.2018

Address for correspondence:

Mina Akbarpour, MD

Student Research Committee

Ahvaz Jundishapur University of Medical Sciences

Ahvaz, Iran

E-mail: jhd.researcher7@gmail.com 